\title{
A Distributed Framework for Supporting 3D Swarming Applications
}

\author{
Ghazaleh Pour Sadrollah*, Jan Carlo Barca*, Asad I. Khan*, Jens Eliasson ${ }^{\dagger}$ and Ilankaikone Senthooran* \\ *Monash Swarm Robotics Laboratory, Faculty of Information Technology, \\ Monash University, VIC, Australia \\ Email: \{ghazaleh.pour.sadrollah, jan.barca, asad.khan, ilankaikone.senthooran\}@monash.edu \\ ${ }^{\dagger}$ EISLAB, Luleå University of Technology, Luleå, Sweden \\ Email: jens.eliasson@1tu.se
}

\begin{abstract}
In-flight wireless sensor networks (WSN) are of increased interest owing to efficiency gains in weight and operational lifetime of IP-enabled computers. High impact 3D swarming applications for such systems include autonomous mapping, surveying, servicing, environmental monitoring and disaster site management. For distributed robotic applications, such as quad copter swarms, it is critical that the robots are able to localise themselves autonomously with respect to other robots and to share information. The importance of fast and reliable dissemination of localised information in these elastic threedimensional networks provides us sufficient reason to present a distributed framework and hardware settings for passing this information pervasively through the swarm. The research field of Internet of Things (IoT) have for several years been addressing issues around low-power, low-bandwidth wireless communication. By applying IoT technologies to the challenges around swarming, new opportunities are created. However, since IoT have been primarily used with stationary devices, the introduction of flying sensors will add more challenges to address.
\end{abstract}

Keywords-Wireless Sensor Networks, Swarm Robotics, Distributed Framework, Internet of Things.

\section{INTRODUCTION}

Swarm Robotics (SR), has drawn increased attention from academics and industry over the last decade. SR has many high-impact real world applications, including autonomous mapping, surveying, servicing, environmental monitoring and disaster site management. In these applications, relative localisation of robots, which operate in unison with peers in close proximity, is a key problem to be solved in order to facilitate efficient coordinated movement and task execution [1][2][3].

The multi-robot systems community has therefore made several attempts at addressing this issue, particularly in the context of SR systems. Relevant works include the: i) omnidirectional vision system presented in [4] that facilitates formation control in groups of nonholonomic ground moving Clodbuster robots, ii) IR based relative positioning module presented in [5] that enable miniature nonholonomic Kephera III robots to maintain formations, and iii) ultrasound based mechanisms presented in [1] which enable centimeter-scale Millibots to leap over large distances while retaining precise position estimates to a reference frame. Another relevant localization platform was presented in [6] where the authors propose a low cost open hardware license board, which enables robots or nodes in sensor networks to determine their relative position and bearing by means of infrared communication.

Furthermore, there have been discussions relevant to $3 \mathrm{D}$ swarm applications, such as the studies in [7] and [8]. The work presented in [7] makes use of an optical motion capture system to track the movement of Micro Air Vehicle (MAV) in 3D space, while [8] presents relative localization requirements for MAVs.

A drawback of the aforementioned works is that the proposed technologies assume that the robots are constrained to a flat 2D surface, are centralised with motion capture systems, have limited sensing distance or have high computational complexity. This assumption may be acceptable for test beds and in laboratory experiment contexts. However, it imposes undesirable constraints on SR systems designed for the real world, as robots must be able to operate in $3 \mathrm{D}$ space to efficiently accomplish tasks in realistic real world scenarios and in a completely decentralised manner.

In this paper, we present a distributed relative localization framework that supports swarming within in-flight sensor networks (swarm of quad copters in this study), which require coordinated movement in 3D space. The proposed framework is therefore ideal for real world SR applications. Quad copters have been selected as the target platform due to their ability to navigate precisely in 3D space and to hover steadily. This technology is also ideal due to its ability to perform vertical takeoff and landing maneuvers, which enables the platform to operate in constrained spaces such as indoor or in rugged terrain where no landing strips are available. The paper also makes a practical contribution by providing a test-bed that will only require open standards based components and software to test distributed consensus and distributed localization algorithms in three dimensions and dynamically. The use of mobile devices in Internet of Things (IoT) has not been well investigated, and the use of standard protocols such as RPL (IPv6 Routing Protocol for Low power and lossy networks) which is mostly designed for stationary networks might not function as designed in highly mobile sensor networks [9]. In this paper, we also investigate the current state of art around research in mobile IoT networks [10], [11].

The proposed relative positioning module utilises wireless communication and message transmission, which require less processing than vision-based systems. The technology is also capable of operating in the dark and is expected to have a significantly longer effective range than the IR, ultrasound and vision-based systems reported in literature.

This paper is outlined as follows; Section II presents the 
proposed framework, followed by Sections III, IV and V which discuss the hardware used by the framework and the proposed network and software design. Finally, conclusions and suggestions for future work are presented in Sections VI and VII, respectively.

\section{PROPOSED FRAMEWORK}

This section describes the distributed relative localization framework for 3D swarming applications of in-flight sensor networks. A schematic overview of the main hardware components and their connection is provided in Figure 1.

The base station enables human operators to interact with the quad copter swarm and primarily functions as a device to monitor and control the overall swarm activity. Furthermore, the quad copter platform represents a node in the swarm that is equipped with a suite of sensor, actuator, communication and processing modules that will be described in greater detail in the following sections.

The main focus of this paper will be on the lightweight low-power Mulle platform [12], which is used for wireless communication, and the open source BeagleBone Black (BBB) board, which handles functional communication-related tasks, and which acts as a central point of connection to all the components on the quad copter [13].

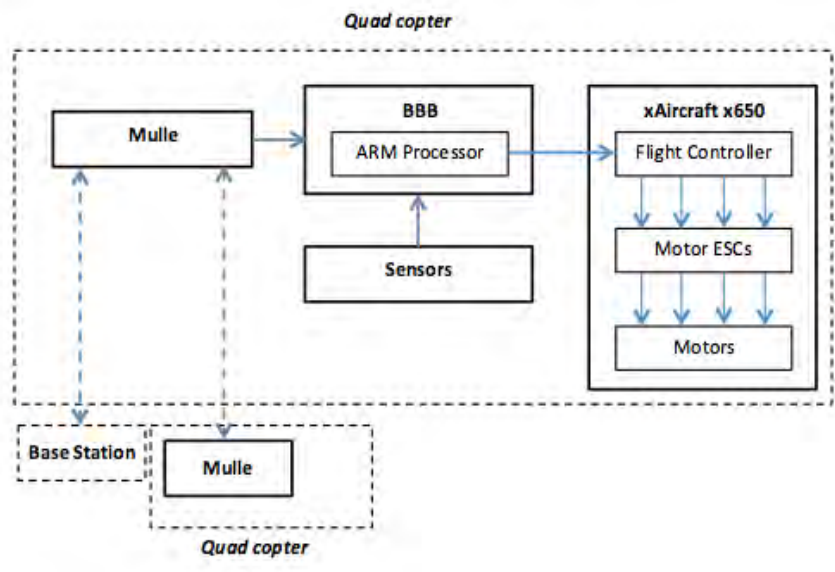

Fig. 1: Main hardware components

\section{HARDWARE DESCRIPTION}

This section provides the details of the various components found in the proposed framework.

\section{A. Quad copter}

The selected XAircraft X650 V8 quad copter platform is illustrated in Fig. 2. This particular quad copter has been selected due to its robust frame, ability to carry relatively large payloads (950 grams) and because it is capable of operating both indoors and outdoors. It is also cost effective when compared to other high-end quad copters such as Datron Scout, Quad Indago and Datron Skyranger.

The Mulle device and the BBB board are stored in the quad copter's fuselage, along with an autopilot, a gyroscope, an accelerometer, and a compass to protect this hardware from

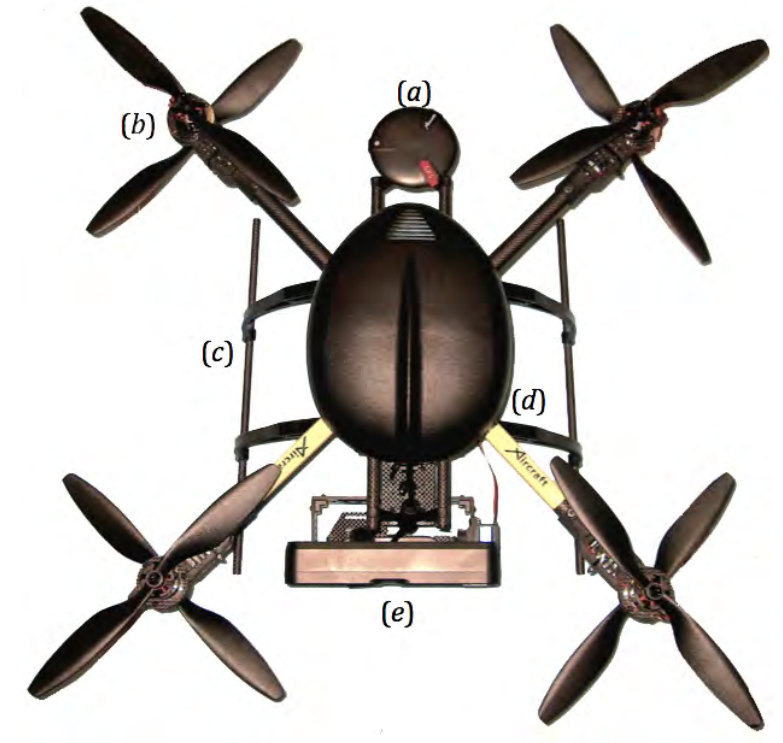

Fig. 2: : (a) Global Positioning System (GPS) module (b) Brushless motor $(c)$ Landing gear $(d)$ Quad copter's fuselage that contains a BeagleBone Black (BBB) board, a Mulle platform, an autopilot, a gyroscope, an accelerometer and a compass (e) Primesense RGB-D sensor

damage, while a Primesense RGB-D sensor is mounted on a gyro stabilized camera mount. The eight brushless motors, which drive the quad copter's propellers, are mounted at the end of cylindrical carbon fibre arms that extend from the main frame. To ensure stable flight, the 11.1 volt battery, which powers the quad copter, is attached at the centre of gravity below the main frame. A Global Positioning System (GPS) module and the landing gear are connected to the quad copter via crossbeams that run through the fuselage. The landing gear is constructed of flexible plastic materials that can withstand impacts upon landing.

\section{B. Mulle}

The Mulle, shown in Figure 3, is a sensor and actuator platform for IoT and WSN applications. The Mulle started as a research project at EISLAB [14], Luleå university of Technology, but is now available commercially from Eistec $\mathrm{AB}$ [12].

The Mulle uses only standardised Internet protocols for communication and features either a Bluetooth 2.0 transceiver or a sub-gigahertz IEEE 802.15.4 radio. The Mulle's small size and low weight of only 3 grams makes it ideal for embedding in swarm applications.

1) Hardware: The Mulle is based on a low-power Renesas M16C/65 microcontroller with $47 \mathrm{kB}$ RAM capable of running at $10 \mathrm{MHz}$. The Mulle features on-board temperature and acceleration sensors, as well as a high-density 60 pin connector for interfacing external sensors and/or actuators. The development environment includes support for the free gcc compiler.

2) Software: The Mulle supports the two most commonly used operating systems for IoT devices: Contiki and TinyOS. The current version of the framework uses the Contiki operating systems which provides a rich set of support for different 


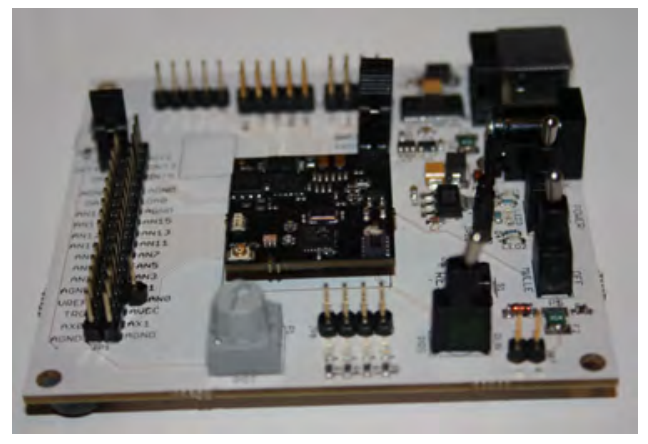

Fig. 3: Mulle v6.2 (black) on development board (white)

sensors, communication protocols, low power system, and other important sub systems.

3) Network: The Mulle's network communication stack provides support for the most commonly used protocols such as as 6LoWPAN, IPv6, CoAP and NTP. Figure 4 shows the protocol stack.

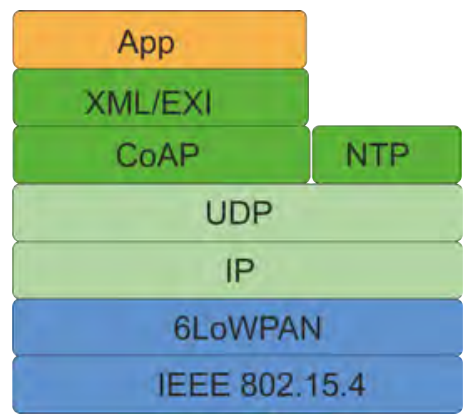

Fig. 4: Mulle Internet of Things network stack

For data representation, the usage of SenML format was chosen. SenML is a structured format developed specifically for sensor applications. The Mulle platform supports SenML with either XML or binary XML (EXI) formats, as demonstrated by Kyusakov et al. [15].

\section{BeagleBone Black}

The BBB board shown in Figure 5, is a communitysupported development platform which features the low-cost Sitara processor developed by Texas Instruments. It is a tiny open-hardware, open-software single-board computer that can be plugged into different devices and computers and can support a variety of applications and tasks, akin to a typical desktop computer.

This particular version of Beagle board has been selected because of its important advantages used in this research, such as its small size, light weight, low power consumption and low cost.

1) Hardware: The BBB has 512MB DDR3 RAM and Micron eMMC on-board flash storage chip that holds up to 2GB of data and allows for 8bit wide access. It also contains embedded 2x PRU 32-bit microcontrollers with I/O and processing power for real-time analysis and a $1 \mathrm{GHz}$ AM335x

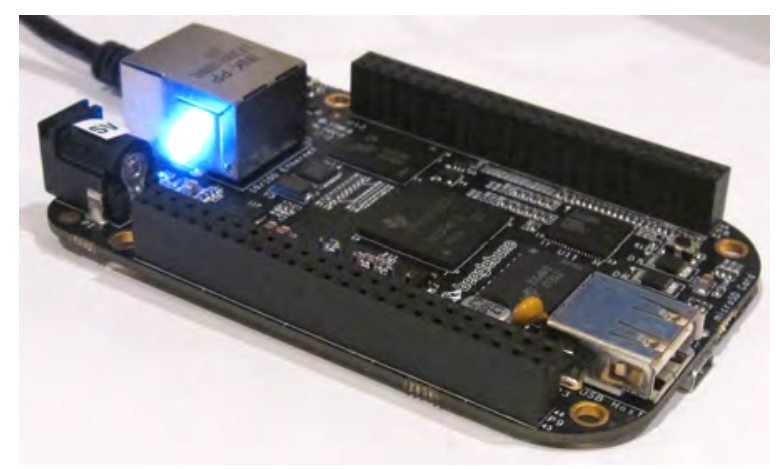

Fig. 5: BeagleBone Black board

ARM Cortex-A8 processor. High-speed peripheral interfaces like USB, Ethernet and HDMI on the credit-card-size BBB mean that these boards can be connected to any alternative device [16].

2) Software: There are four boot modes on BBB which are eMMC Boot that is a default mode, SD Boot which is boot from SD card, Serial Boot that is a serial port to allow downloading and USB Boot which is over a USB port [17]. The BBB runs and supports various flavours of the Linux operating system such as Angstrom, Fedora and Ubuntu, thus enabling re-use of code and application which greatly reduces development time. The BBB also supports HDMI output so it can be used as a standalone computer too.

\section{Proposed Network Architecture}

A Mulle running Contiki's RPL border router gateway software is used to interface the quad copter's BBB device. The Mulle can therefor be used as a wireless interface with long range capabilities. The $868 \mathrm{MHz}$ Mulle v6.2 has a range of more than $1 \mathrm{~km}$ when operating with line of sight. Any sensor attached to the Mulle is also accessible from the Beaglebone using the CoAP protocol. The architecture is based on usage of multiple mobile gateways in the same network, as discussed by Silva et al. [18], which put constraints on routing tables and IPv6 prefix use.

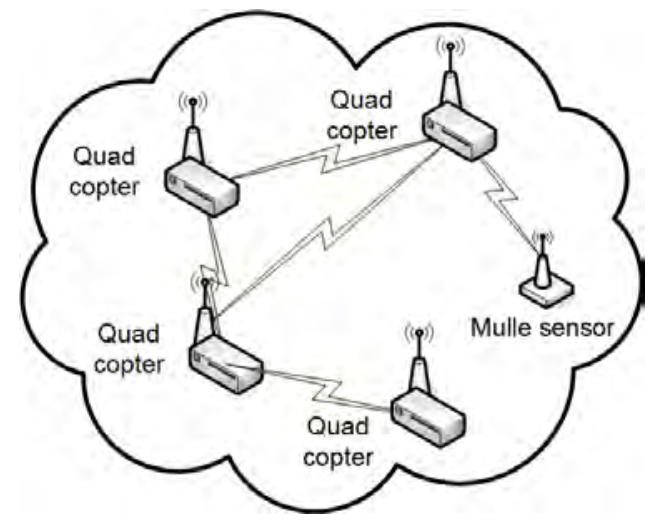

Fig. 6: Network architecture

In Fig. 6, the proposed network architecture is shown. 
The use of low-power and long-range wireless communication enables a swarm to stay connected when flying. The current setup is based on Contiki 2.7 RPL border router software running on a BBB platform. In [19], Petajajarvi et al. investigated the performance of mobility in IoT networks using 6LoWPAN. More work is however needed to fully support a mobile network composed of mobile sensors and gateways.

\section{Proposed Software Design}

In this section the software design is proposed along with important applications and software used for communication. The BBB supports different operating systems such as Angstrom Linux, Fedora and Ubuntu. As discussed in the previous section, the default boot mode for the BBB boards is MMC1 with an option to change it to MMC0 for SD card booting. The boards are pre-configured with Angstrom operating system. In this study we installed a new Ubuntu image on the boards by using SD Boot mode due to its large available support community and relevant packages that enable us to use a variety of programming languages such as Java and C.

For the BBB boards to communicate effectively and share information, a command line cluster is built that would be controlled via a laptop or desktop/base station. Secure Shell (SSH) which is a cryptographic network protocol for secure data communication, is selected to let BBB boards have a remote login to the other boards and machines that are connected via a wireless network provided by Mulle.

For passing messages between quad copters in a network, Message Passing Interface (MPI) is selected. The advantages of developing message-passing software using MPI closely match the design goals of efficiency, speed, flexibility and portability, as it is almost universally compatible with almost every distributed architecture. Furthermore, it is supporting several languages such as C, Python and Java [20].

There are different versions of MPI available. In this study MPICH is chosen. MPI uses SSH to connect to the other boards and a shared folder to run and share the MPI code with all the nodes within the cluster. After establishing a connection among all the boards, MPI code is utilised to allow the robots to send and receive data and information asynchronously within the swarm [21].

\section{CONCLUSION}

In this paper, a distributed localisation framework for supporting 3D swarming applications is presented. A general hardware setting for controlling swarms of in-flight sensor networks is proposed by combining IoT technologies with swarm robotics.

Furthermore, this project aims to develop novel interaction technology to maintain control, localisation and coordination between the swarm of quad copters and a human operator located at a base station. The next steps consist of providing a full conceptual design to human - swarm interaction and further investigating a suitable consensus algorithm. The physical and operational experiments are being designed and conducted in order to test and validate the proposed system.

\section{FUTURE WORK}

The use of the RPL protocol on mobile devices must be further investigated as suggested by [22]. Furthermore, the use of the Mulle platform for 3D swarm localisation must also be investigated further. Previous work shows that the Mulle's IEEE 802.15.4 radio can be used for course grain localisation, and newer radio chips with time-of-flight distance measurement capabilities can enhance the accuracy.

Moreover, as discussed in [23], the functionality of quad copters over large open areas, and supporting the power of necessary components such as BBB boards, cameras and Mulle, require repeated recharging of the on-board battery, which needs to be discussed at greater length. Finally, agreement mechanisms that can propagate control commands through the swarm in a distributed fashion must also be devised to ensure that human operators can interact with the swarm in a manner that does not violate the principles of SR.

\section{ACKNOWLEDGMENT}

The authors would like to thank The Swedish Foundation for International Cooperation in Research and Higher Education (STINT), institutional grant IG2011-2025 for funding this research.

The authors would also like to express their gratitude towards our partners within the Arrowhead project, and the European commission and Artemis for funding.

\section{REFERENCES}

[1] L. E. Navarro-serment, C. J. Paredis, and P. K. Khosla, "A beacon system for the localization of distributed robotic teams," in In Proceedings of the International Conference on Field and Service Robotics, 1999, pp. 232-237.

[2] J. Barca and R. Koon Li, "Augmenting the human entity through man/machine collaboration," in Computational Cybernetics, 2006. ICCC 2006. IEEE International Conference on, 2006, pp. 1-6.

[3] J. Barca and Y. A. Sekercioglu, "Swarm robotics reviewed," pp. 345359, 2013.

[4] A. Das, R. Fierro, V. Kumar, J. Ostrowski, J. Spletzer, and C. Taylor, “A vision-based formation control framework," Robotics and Automation, IEEE Transactions on, vol. 18, no. 5, pp. 813-825, 2002.

[5] J. Pugh, X. Raemy, C. Favre, R. Falconi, and A. Martinoli, "A fast onboard relative positioning module for multirobot systems," Mechatronics, IEEE/ASME Transactions on, vol. 14, no. 2, pp. 151-162, 2009.

[6] A. Gutierez, A. Campo, D. Amor, L. Magdalena, and F. MonasterioHeulin, "An open localization and local communication embodied sensor," Sensors, 2008.

[7] N. Michael, D. Mellinger, Q. Lindsey, and V. Kumar, "The grasp multiple micro-uav testbed." IEEE Robot. Automat. Mag., vol. 17, no. 3, pp. 56-65, 2010. [Online]. Available: http://dblp.unitrier.de/db/journals/ram/ram17.html\#MichaelMLK10

[8] J. Faigl, T. Krajnk, J. Chudoba, L. Preucil, and M. Saska, "Low-cost embedded system for relative localization in robotic swarms." in ICRA. IEEE, 2013, pp. 993-998. [Online]. Available: http://dblp.uni-trier.de/db/conf/icra/icra2013.html\#FaiglKCPS13

[9] V. Safdar, F. Bashir, Z. Hamid, H. Afzal, and J. Y. Pyun, "A hybrid routing protocol for wireless sensor networks with mobile sinks," in Wireless and Pervasive Computing (ISWPC), 2012 7th International Symposium on, July 2012, pp. 1-5.

[10] L. Deru, S. Dawans, M. Ocaa, B. Quoitin, and O. Bonaventure, "Redundant border routers for mission-critical 6lowpan networks," in Proceedings of the Fifth Workshop on Real-World Wireless Sensor Networks, 2013.

[11] A. Jara, M. Zamora, and A. Skarmeta, "Intra-mobility for hospital wireless sensor networks based on 6lowpan," in Wireless and Mobile Communications (ICWMC), 2010 6th International Conference on, Sept 2010, pp. 389-394.

[12] “Eistec AB," Eistec AB, 2014, http://www.eistec.se/. 
[13] D. Ricart. (2013, Nov.) Building a compute cluster with the beaglebone black. [Online]. Available: https://www.linux.com/community/blogs/133-generallinux/748871-building-a-compute-cluster-with-the-beaglebone-black

[14] "Luleå University of Technology, Embedded Internet System Laboratory," Jan 2014, http://www.ltu.se/eislab.

[15] R. Kyusakov, H. Makitaavola, J. Delsing, and J. Eliasson, "Efficient $\mathrm{xml}$ interchange in factory automation systems," in IECON 2011 - 37th Annual Conference on IEEE Industrial Electronics Society, 2011, pp. $4478-4483$.

[16] J. Kridner and T. Stratton. (2013, Nov.) What is beaglebon black? [Online]. Available: http://beagleboard.org/Products/beaglebone

[17] G. Coley and P. J. R. Day. (2013, Nov.) Beaglebone black system reference manual.

[18] R. Silva, J. Sa Silva, M. Simek, and F. Boavida, "A new approach for multi-sink environments in wsns," in Integrated Network Management, 2009. IM '09. IFIP/IEEE International Symposium on, June 2009, pp. 109-112.

[19] J. Petajajarvi and H. Karvonen, "Soft handover method for mobile wireless sensor networks based on 6lowpan," in Distributed Computing in Sensor Systems and Workshops (DCOSS), 2011 International Conference on, June 2011, pp. 1-6.

[20] "Message passing interface," International Journal of High Performance Computing Applications, 1993.

[21] B. Barney, "Message passing interface (mpi)," Lawrence Livermore National Laboratory.

[22] K. Heurtefeux, H. Menouar, and N. AbuAli, "Experimental evaluation of a routing protocol for wsns: Rpl robustness under study," in Wireless and Mobile Computing, Networking and Communications (WiMob), 2013 IEEE 9th International Conference on, 2013, pp. 491-498.

[23] K. Dantu, S. Berman, B. Kate, and R. Nagpal, "A comparison of deterministic and stochastic approaches for allocating spatially dependent tasks in micro-aerial vehicle collectives," in Intelligent Robots and Systems (IROS), 2012 IEEE/RSJ International Conference on, 2012, pp. 793-800. 